ARTICLE

DOI: $10.1038 / \mathrm{s} 41467-018-02827-7$

\title{
Crystal structure reveals vaccine elicited bactericidal human antibody targeting a conserved epitope on meningococcal fHbp
}

\author{
Jacinto López-Sagaseta ${ }^{1}$, Peter T. Beernink (iD ${ }^{2}$, Federica Bianchi ${ }^{1}$, Laura Santini ${ }^{1}$, Elisabetta Frigimelica (1) ${ }^{1}$,
} Alexander H. Lucas², Mariagrazia Pizza ${ }^{1} \&$ Matthew J. Bottomley ${ }^{3}$

Data obtained recently in the United Kingdom following a nationwide infant immunization program against serogroup B Neisseria meningitidis (MenB) reported $>80 \% 4 C M e n B$ vaccinemediated protection. Factor $\mathrm{H}$-binding protein ( $\mathrm{fHbp}$ ) is a meningococcal virulence factor and a component of two new MenB vaccines. Here, we investigated the structural bases underlying the $\mathrm{fHbp}$-dependent protective antibody response in humans, which might inform future antigen design efforts. We present the co-crystal structure of a human antibody Fab targeting fHbp. The vaccine-elicited Fab $1 \mathrm{~A} 12$ is cross-reactive and targets an epitope highly conserved across the repertoire of three naturally occurring fHbp variants. The free Fab structure highlights conformational rearrangements occurring upon antigen binding. Importantly, 1A12 is bactericidal against MenB strains expressing fHbp from all three variants. Our results reveal important immunological features potentially contributing to the broad protection conferred by $\mathrm{fHbp}$ vaccination. Our studies fuel the rationale of presenting conserved protein epitopes when developing broadly protective vaccines.

\footnotetext{
${ }^{1}$ GSK Vaccines srl, Via Fiorentina 1, 53100 Siena, Italy. ${ }^{2}$ Immunobiology and Vaccine Development, UCSF Benioff Children's Hospital, 5700 Martin Luther King Jr. Way, Oakland, CA 94609, USA. ${ }^{3}$ GSK Vaccines, 14200 Shady Grove Road, Rockville, MD 20817, USA. Alexander H. Lucas is deceased. Correspondence and requests for materials should be addressed to J.L.-S. (email: jlopez.sagaseta@gmail.com) or to M.J.B. (email: matthew.j.bottomley@gsk.com)
} 
M eningococci cause fatal cases of bacterial sepsis and meningitis, with serogroup B (MenB) strains particularly prevalent in Europe ${ }^{1,2}$. Two vaccines based on protein antigens were developed for the prevention of MenB disease. One of these antigens is factor H-binding protein ( $\mathrm{fHbp}$ ), which was identified independently by reverse vaccinology using genomic sequences ${ }^{3}$ and by traditional methods using biochemical fractionation ${ }^{4}$. FHbp elicits protective antibody responses in mice, rabbits, rhesus macaques ${ }^{3,5,6}$, and humans ${ }^{7-9}$. The vaccines are referred to as 4CMenB (Bexsero; GSK) and Bivalent rLP2086 (Trumenba; Pfizer) and both are licensed for use in adolescents in the United States. Only $4 \mathrm{CMenB}$ is licensed for infants starting 2 months of age in Europe, Canada, Australia, and several countries in South America. Of note, following a nationwide implementation of $4 \mathrm{CMenB}$, a recent study showed $>80 \%$ vaccine-mediated protection against all current MenB strains in the United Kingdom ${ }^{10,11}$.

Antibodies to $\mathrm{fHbp}$ elicit protection through complementmediated bactericidal activity ${ }^{3,4}$. Some antibodies also inhibit the binding of human complement factor $\mathrm{H}(\mathrm{fH})$ to the bacteria, rendering them more susceptible to complement ${ }^{12}$. While some antibodies to $\mathrm{fHbp}$ elicited in mice inhibited the binding of $\mathrm{fH}$ to the bacterial surface ${ }^{12,13}$, the antibodies elicited in rhesus macaques $^{14,15}$ or humans ${ }^{16}$ generally did not inhibit binding of $\mathrm{fH}$. This difference may result from the inability of murine $\mathrm{fH}$ to bind $\mathrm{fHbp}^{16}$, in contrast to human $\mathrm{fH}$ that binds $\mathrm{fHbp}$, such that the dynamics of epitope exposure, dependent on $\mathrm{fH}$ binding, are likely different when immunizing mice and humans.

Bactericidal polyclonal antibodies raised in mice were reported to be mainly directed against the carboxyl (C)-terminal domain of $\mathrm{fHbp}^{17}$. Epitope mapping of murine anti-fHbp monoclonal antibodies (mAbs) has confirmed that many of the amino-acid residues involved in antibody binding are located in the $\mathrm{C}$ terminal domain ${ }^{17-19}$. There are several examples, however, of epitopes involving residues in the amino $(\mathrm{N})$-terminal domain ${ }^{20-}$ 23 . Detailed epitope-mapping studies of anti-fHbp mAbs have been performed using nuclear magnetic resonance spectroscopy ${ }^{18,22}$, hydrogen-deuterium exchange followed by mass spectrometry ${ }^{21,24}$, and by X-ray crystallography ${ }^{24,25}$. The latter studies recently defined a mechanism by which two murine antifHbp antibodies (mAbs JAR5 and 12C1) may synergize to elicit complement-mediated bactericidal activity ${ }^{25,26}$. Moreover, both $\mathrm{mAbs}$ target epitopes that overlap with the $\mathrm{fH}$-binding site ${ }^{24,25}$, thus revealing the structural basis for their inhibition of $\mathrm{fH}$ binding. Structural epitope-mapping studies with murine Fabs have also been performed for another protective antigen present in $4 \mathrm{CMenB}$, namely the outer membrane protein PorA ${ }^{27-29}$.

In an important recent study, the human antibody repertoire to $\mathrm{fHbp}$ was investigated for the first time, by characterization of a panel of 10 human anti-fHbp antibody fragments (Fabs) cloned from three subjects vaccinated with $4 \mathrm{CMenB}^{16}$. Therein, two of the three subjects raised broadly reactive antibodies (termed $9 \mathrm{~B}$ and 10C). Fab 9B (hereafter termed Fab 1A12) was of particular interest since it bound with extremely high affinity $\left(K_{\mathrm{D}}=19 \mathrm{pM}\right)$ to fHbp variant 1.1 (var1.1) and, moreover, cross-reacted with all eight fHbp sequence variants tested, including representatives from all three phylogenetic variant groups. This Fab was particularly unusual because most known antibodies against fHbp are "variant group-specific", i.e., most mAbs efficiently bind fHbp from one variant group, but not from both the other two variant groups. Indeed, despite previous analyses of hundreds of mAbs raised against fHbp by animal immunizations, only a few have been reported to exhibit some cross-reactivity, including MN86994- $11^{30}, \mathrm{JAR} 41^{23}, 17 \mathrm{C} 1^{21}$, and $30 \mathrm{G} 4^{21}$. Within the fHbp variant groups, amino-acid sequence identity is usually above $87 \%$; whereas, between variant groups the sequence identity can fall to as little as $62 \%$, and this high antigenic variability presumably underlies the rarity of eliciting cross-reactive mAbs $3,23,30$.

The observations summarized above raise the question: "What is the structural basis of the broad antigen-recognition properties of the vaccine-elicited human antibody 1A12?" Since meningococci display enormous antigenic diversity $(\sim 1000$ sequence variants of $\mathrm{fHbp}$ have been reported ${ }^{31}$ ), it is important to understand how current MenB vaccine antigens interact with the human immune system. Such details are expected to provide insights into vaccine efficacy and may enable the design of nextgeneration vaccines.

In this study, we present the crystal structures of the broadly reactive Fab $1 \mathrm{~A} 12$ alone and in a complex with $\mathrm{fHbp}$, thereby elucidating the structural basis for the antigen-recognition properties of this human antibody. We also show that Fab $1 \mathrm{~A} 12$ as an intact IgG antibody has high affinity for different fHbp variants, and for point mutants, revealing the contribution of specific amino acids in the epitope recognized by the human antibody. Finally, in functional assays, IgG $1 \mathrm{~A} 12$ has bactericidal activity. These data provide the crystallographic and functional characterization of a functional vaccine-elicited human antibody targeting a bacterial pathogen.

\section{Results}

Human mAb 1 A12 shows affinity and broad reactivity for fHbp. Fab 1A12 derives from an adult human subject immunized with a MenB vaccine formulation that contained fHbp var1.1 (see Methods). The cross-reactivity of recombinant Fab $1 \mathrm{~A} 12$ in enzyme-linked immunosorbent assay (ELISA) experiments using the three different variant groups of $\mathrm{fHbp}$ was reported previously $^{16}$. To extend those investigations, here we used mammalian cells to produce $1 \mathrm{~A} 12$ as an intact full-length mAb of the IgG1 subclass (the subclass most abundant in human sera), and Escherichia coli to produce recombinant $\mathrm{fHbp}$ antigens. Surface plasmon resonance (SPR) was used to determine the kinetics for immobilized mAb $1 \mathrm{~A} 12$ binding to solution phase fHbp antigens representative of the three different variant groups: fHbp var1.1; fHbp var2.16; and fHbp var3.45. All three variants were recognized by $\mathrm{mAb} 1 \mathrm{~A} 12$, as indicated by the sub-nanomolar equilibrium dissociation constant $\left(K_{\mathrm{D}}\right)$ values of 87,384 , and $138 \mathrm{pM}$ for fHbp var1.1, var2.16, and var3.45, respectively (Fig. 1 and Table 1).

Table 1 Binding kinetic values determined for mAb $1 A 12$ by surface plasmon resonance

\begin{tabular}{|c|c|c|c|c|c|c|c|c|c|c|}
\hline & Var1.1 & Var2.16 & Var3.45 & Var1.1 A162P & Var1.1 G163A & Var1.1 G163N & Var1.1 K180A & Var1.1 K185A & Var1.1 N190A & Var1.1 N215G \\
\hline $\begin{array}{l}k_{\text {on }}\left(M^{-1} s^{-1}\right) \times 10^{5} \\
k_{\text {off }}\left(s^{-1}\right) \times 10^{-5} \\
K_{D}{ }^{a}(p M)\end{array}$ & $\begin{array}{l}6.2 \pm 0.1 \\
5.4 \pm 0.7 \\
87 \pm 10\end{array}$ & $\begin{array}{l}2.3 \pm 0.01 \\
8.7 \pm 0.5 \\
384 \pm 24\end{array}$ & $\begin{array}{l}4.2 \pm 0.01 \\
5.7 \pm 0.3 \\
138 \pm 7\end{array}$ & $\begin{array}{l}10.1 \pm 0.8 \\
2.4 . \pm 0.9 \\
24 \pm 11\end{array}$ & $\begin{array}{l}6.3 \pm 0.02 \\
2.7 \pm 0.2 \\
44 \pm 3\end{array}$ & $\begin{array}{l}8.3 \pm 1.0 \\
4.6 \pm 0.7 \\
55 \pm 2\end{array}$ & $\begin{array}{l}3.3 \pm 0.01 \\
0.9 \pm 0.2 \\
28 \pm 6\end{array}$ & $\begin{array}{l}1.5 \pm 0.02 \\
32.1 \pm 1.8 \\
2158 \pm 149\end{array}$ & $\begin{array}{l}4.7 \pm 0.2 \\
175.8 \pm 7.9 \\
3713 \pm 11\end{array}$ & $\begin{array}{l}8.1 \pm 0.04 \\
50.2 \pm 0.7 \\
620 \pm 12\end{array}$ \\
\hline
\end{tabular}



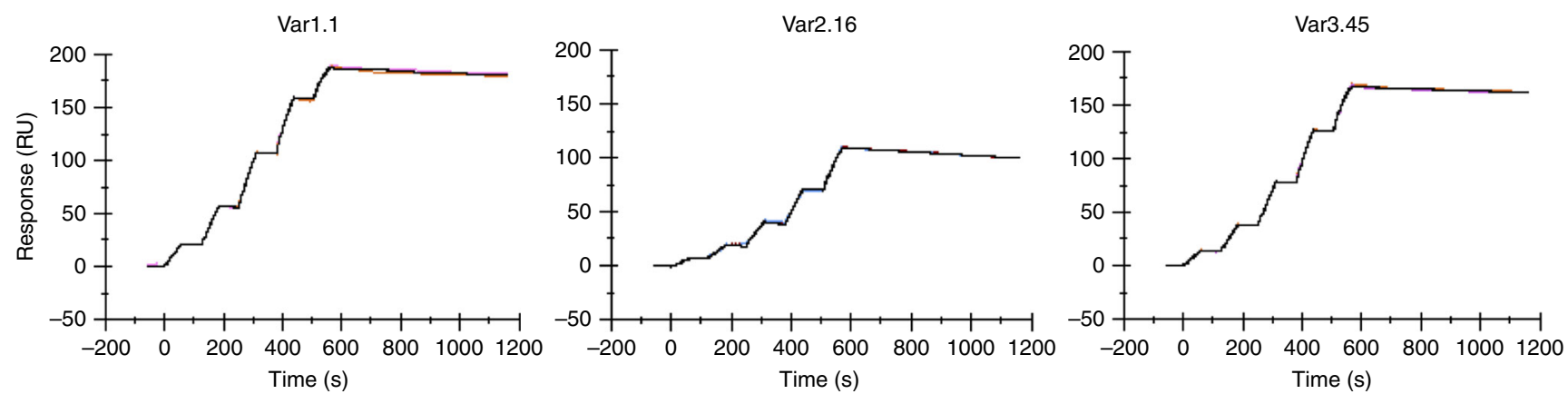

Fig. $1 \mathrm{mAb} 1 \mathrm{~A} 12$ shows high-affinity cross-reactive binding to fHbp in SPR studies. In each panel, sensorgrams show the experimental association and dissociation traces (colored) performed in duplicate for the binding of the different fHbp subvariants to captured mAb 1A12; the calculated fitting traces are shown in dark gray. Full kinetic analyses of each interaction are reported in Table 1

Structure determination of human Fab 1 A12 bound to fHbp. Since mAb $1 \mathrm{~A} 12$ was raised by vaccination with fHbp var1.1, we sought structural information to explain its cross-reactivity and the precise recognition mode of its epitope. We obtained crystals of Fab $1 \mathrm{~A} 12$ bound to fHbp var1.1 that initially diffracted to $3.5 \AA$ resolution. By an iterative streak-seeding approach, we subsequently obtained better diffracting crystals (belonging to space group $\mathrm{P} 2_{1}$ ) and ultimately determined the structure via molecular replacement with a resolution of $2.2 \AA\left(I / \sigma I=0.98, C^{1 / 2}=0.26\right.$ in the highest resolution shell ${ }^{32}$, see Methods and Table 2). Two $\mathrm{Fab} / \mathrm{fHbp}$ complexes were present in the asymmetric unit and were essentially identical, exhibiting a root mean square deviation (rmsd) of $0.5 \AA$ across all alpha carbon atoms. The overall structure of the complex shows Fab $1 \mathrm{~A} 12$ projecting all six complementarity-determining region (CDR) loops onto a surface-exposed region at one end of the C-terminal $\beta$ barrel of $\mathrm{fHbp}$, while the N-terminal region of fHbp does not contribute to the interaction (Fig. 2). Overall, $17 \mathrm{fHbp}$ residues are involved in a curved interface. The buried surface area on fHbp is $800 \AA^{2}$, which is typical for Fab/antigen complexes ${ }^{33,34}$. Fab $1 \mathrm{~A} 12$ binds fHbp with a major contribution from the heavy chain, and a minor contribution from the light chain $\left(590 \AA^{2}\right.$ vs. $\left.210 \AA^{2}\right)$. The binding interface comprises charged, polar, and van der Waals (VDW) interactions.

The Fab 1A12-binding site on fHbp is completely different from the two structurally characterized epitopes of the murine Fabs $12 \mathrm{C} 1$ and JAR5 ${ }^{24,25}$, which are both specific only for fHbp variant group 1 antigens. To compare the modes of binding to fHbp, we conceptually divided the fHbp molecule into quadrants by drawing "crosshairs" on its long and short axes, thus creating a reference frame (Fig. 3). While both JAR5 and 12C1 target the left half of fHbp, and in particular the upper (N-terminal) and lower (C-terminal) quadrants, respectively, 1A12 binds fHbp on its lower right quadrant, in a distinctly new region (Fig. 3). Similarly, the 1A12-binding site does not overlap that of human factor $\mathrm{H}$, which binds on the two left quadrants of $\mathrm{fHbp}^{35}$, thus providing the molecular explanation for previous observations that Fab $1 \mathrm{~A} 12$ does not inhibit binding of fHbp to factor $\mathrm{H}^{16}$.

Details of a cross-reactive conformational epitope on fHbp. A close inspection of the Fab 1A12/fHbp-binding interface reveals a predominant role in antigen recognition for the Fab heavy chain, and especially for the heavy chain variable $\left(\mathrm{V}_{\mathrm{H}}\right)$ CDR3 loop which extends into a notable groove on the fHbp surface (Fig. 4a). In the $\mathrm{V}_{\mathrm{H}}$ CDR3 loop, all residues from Q101 to P107 (except V102) act to secure an extensive network of backbone and sidechain polar and VDW contacts, and presumably all contribute to the extremely tight interaction with the antigen (Fig. $4 \mathrm{a}$ and
Table 2 X-ray data collection, processing, and refinement statistics

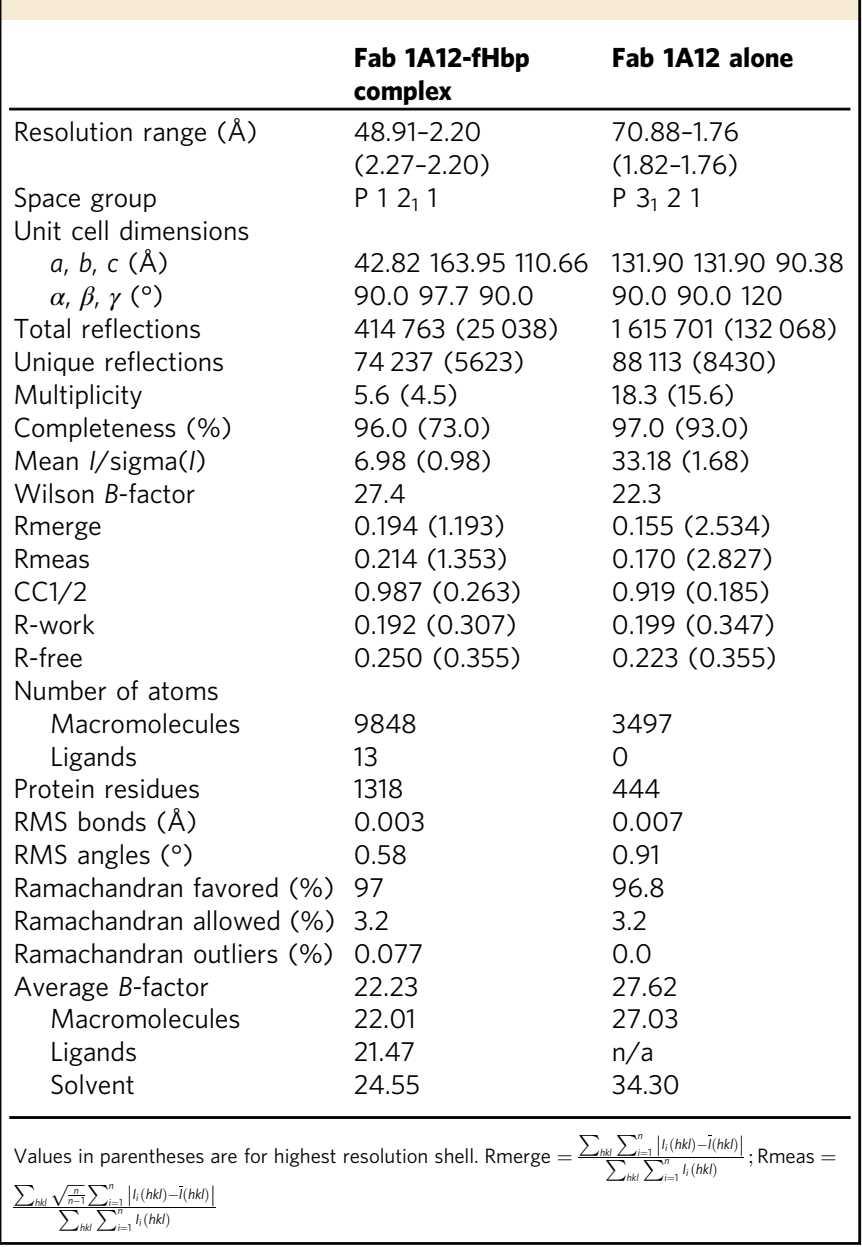

Supplementary Table 1). In addition, several other striking contacts are established via salt bridges between Asp161 on fHbp and Arg54 on the heavy chain (Fig. 4b, upper left), and Lys 185 on fHbp and Asp55/Asp57 on the heavy chain (Fig. 4b, lower left), and, via hydrogen bonds between Asn190 on fHbp and Gln101 on $\mathrm{V}_{\mathrm{H}} \mathrm{CDR} 3$ (Fig. $4 \mathrm{~b}$, upper right). Further, a water-mediated hydrogen bond is formed between Thr91 in the light chain CDR3 and Tyr214 on fHbp (Fig. 4b, lower right). Importantly, Asn215 on fHbp simultaneously contacts both the heavy and light chains of $\mathrm{Fab} 1 \mathrm{~A} 12$, by hydrogen bonding with the gamma oxygen 


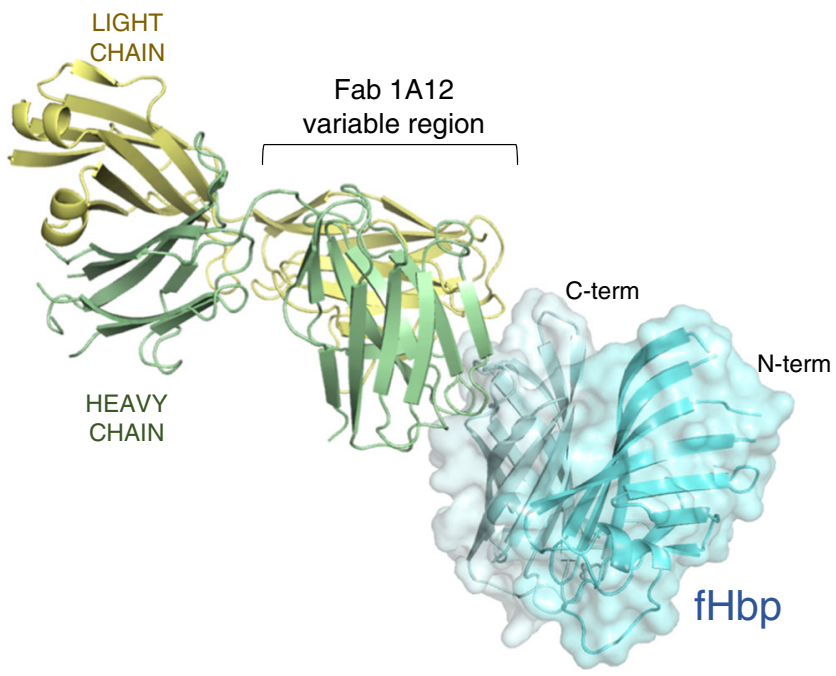

Fig. 2 The Fab 1A12-fHbp complex crystal structure. Ribbon diagram in which the heavy and light chains of Fab 1A12 are colored green and yellow, respectively; fHbp is represented in cyan with a transparent surface. Artwork was prepared using PyMOL

atoms of three serine residues (heavy chain Ser106 directly, and light chain Ser30 and Ser32 indirectly through water-mediated interactions) and with Val31 (backbone nitrogen) on the light chain (Fig. 4c).

A surface representation of all the $\mathrm{fHbp}$ residues that interact with $1 \mathrm{~A} 12$ reveals the nature of the conformational epitope on $\mathrm{fHbp}$, lying on a surface-exposed well-ordered region of the Cterminal $\beta$ barrel. The epitope is concentrated in a cluster of residues targeted by the $\mathrm{V}_{\mathrm{H}}$ CDR2 and CDR3 loops, and a more isolated area contacted by the light chain (Fig. 5a).

Basis of 1 A12 cross-reactivity despite antigenic diversity. The elucidation of the present structure allows us to provide a detailed molecular explanation for the versatility of mAb $1 \mathrm{~A} 12$ to recognize fHbp antigens from all three variant groups. Remarkably, many of the fHbp residues that participate in the interaction with the Fab ( 12 of the 17 residues in the $1 \mathrm{~A} 12$ epitope) are conserved across the three different fHbp variants tested here by SPR, i.e., var1.1, var2.16, and var3.45 (Fig. 5b). Notably, residues Asp161 and Asn190 are completely conserved in fHbp variants 1.1, 2.16, and 3.45, and play key roles in the overall network of interactions with the Fab (Fig. 4b). Further, the motif ${ }_{180} \mathrm{KIEHLK}_{185}$, and residues Asn190, Val191, and Tyr214 are also conserved in the same three variants tested by SPR. Therefore, the degree of conservation assigns a leading role to these residues in the crossrecognition by the human $\mathrm{mAb} 1 \mathrm{~A} 12$.

The Neisseria Multi Locus Sequence Typing database now contains $\sim 1000$ different polypeptide sequences for fHbp obtained from naturally occurring strains ${ }^{31}$. Therefore, we performed a deeper analysis in silico and calculated the degree of conservation associated with residues in the $1 \mathrm{~A} 12$ epitope in 984 fHbp sequence variants available to date, which include sequences from serogroup B strains and from other serogroups ${ }^{31}$. Most notably, five residues (Ile181, Glu182, Leu184, Val191, and Tyr214) are $100 \%$ conserved throughout the whole fHbp sequence repertoire (Fig. 5b). Furthermore, five additional epitope residues show $\geq 99 \%$ conservation (Asp161, His183, Lys185, Pro187, and Asn190). Together, these observations suggest that mAb 1A12 might display cross-reactivity with a vast breadth of recognition across almost the entire known polymorphic repertoire of $\mathrm{fHbp}$.
Effects of polymorphisms in the 1A12-fHbp-binding interface. To better define the contribution of individual residues and the effect of polymorphisms within the epitope, we made singleamino-acid substitutions in the fHbp var1.1 background: A162P; G163A; G163N; K180A; K185A; N190A; and N215G. The fHbp Asn 215 residue makes contacts directly or indirectly with six different residues in the heavy and light chains of Fab $1 \mathrm{~A} 12$ (Fig. 4c and Supplementary Table 1). Substitution of Asn215 with Gly $(\mathrm{N} 215 \mathrm{G})$, as found in var2.16 and var3.45, resulted in a notable decrease in binding to mAb $1 \mathrm{~A} 12$ (Fig. 6a). However, given the extremely tight binding of mAb $1 \mathrm{~A} 12$ to wild-type var1.1, the seven-fold decrease in affinity due to the $\mathrm{N} 215 \mathrm{G}$ mutation nevertheless resulted in a very tight antigen/antibody interaction $\left(K_{\mathrm{D}}=620 \mathrm{pM}\right.$, compared to $87 \mathrm{pM}$ for wild type).

The fHbp residue Gly163 is also found in the epitope/paratope interface, using its backbone carbonyl oxygen to contact the important heavy chain residue Arg54 (Fig. 4b). It was conceivable that this backbone-mediated interaction coupled with the small size of Gly163 (and of its neighbor Ala162) were key factors in the optimal accommodation of the Arg54 side chain from 1A12. However, the single mutations A162P and G163N (both polymorphisms naturally occurring at this position in some variant 2 and 3 proteins) actually resulted in mild increases in binding affinity toward mAb $1 \mathrm{~A} 12$ (Fig. 6b, c). fHbp var1.1 G163A and K180A mutants also slightly increased the binding affinity (approximately two- to threefold), thus suggesting that the side-chain characteristics in these positions are not crucial determinants for cross-recognition by mAb $1 \mathrm{~A} 12$ (Table 1 and Fig. $6 \mathrm{~d}, \mathrm{e})$. These results contribute to a better understanding of the molecular basis of this interaction and the potential overall cross-reactivity of mAb 1A12, since G163 and K180 show lower prevalence throughout all strains (G163 is found in $~ 50 \%$ of subvariants; K180 in $~ 70 \%$ of subvariants), despite both being conserved in var1.1, var2.16, and var3.45. In summary, known polymorphisms occurring in fHbp at positions 163 and 180 are unlikely to preclude binding of mAb $1 \mathrm{~A} 12$ to meningococcal strains expressing such antigens.

Finally, we studied the roles of Lys185 and Asn190. The former establishes salt bridges with two acidic residues on the Fab heavy chain, Asp55 and Asp57, and VDW contacts with His52 and Arg54 (Fig. 4b and Supplementary Table 1) while the latter is directly involved in hydrogen bonds with Gln101 and Gly104 and VDW contacts with Ser103. In SPR studies, mutant K185A reduced the binding affinity to $1 \mathrm{~A} 12$ by $\sim 25$-fold (Fig. $6 \mathrm{f}$ and Table 1). Similarly, N190A notably impacted the binding to $1 \mathrm{~A} 12$ with an $\sim 50$-fold reduction in the affinity (Fig. $6 \mathrm{~g}$ and Table 1 ). While both the association and dissociation rates are affected in the case of $\mathrm{K} 185 \mathrm{~A}$, it is predominantly the increased dissociation rate $\left(k_{\text {off }}\right)$ that is responsible for the lower affinity of N190A. These results confirm the importance of these highly conserved positions on $\mathrm{fHbp}$ recognition by $1 \mathrm{~A} 12$.

In conclusion, the very high affinity of mAb $1 \mathrm{~A} 12$ for wild-type fHbp var1.1 appears to be the result of a cooperative and elaborate network of interactions. Despite the sequence diversity inherent to $\mathrm{fHbp}$, we show here that $\mathrm{mAb} 1 \mathrm{~A} 12$ recognizes $\mathrm{a}$ series of fHbp variants with very high affinities, suggesting a high breadth of coverage potentially conferred by this human $\mathrm{mAb}$.

Structure of free Fab $1 \mathrm{A1} 2$ reveals paratope flexibility. We also determined the crystal structure of the free Fab $1 \mathrm{~A} 12$ at $1.76 \AA$ resolution (Table 2). Comparison of the free and antigen-bound Fab structures shows that they are highly similar (rmsd $0.69 \AA$ on alpha carbons). However, superposition reveals that while most of the CDR loops do not change their conformation (Fig. 7a), there 

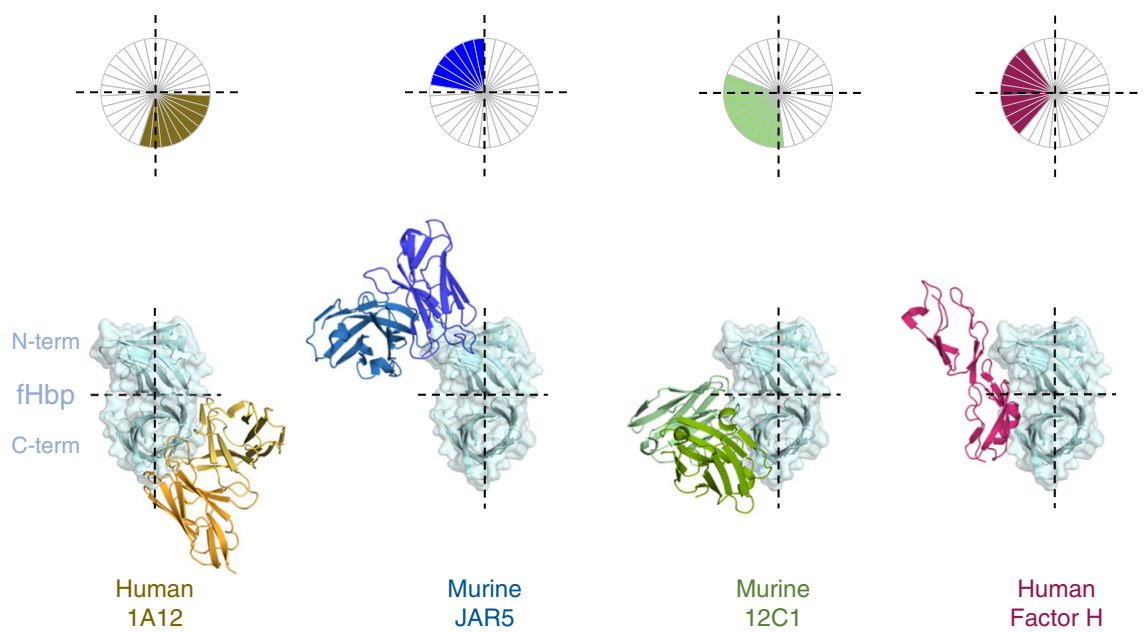

Fig. 3 Fab $1 \mathrm{~A} 12$ shows a unique binding mode. Bottom: surface and ribbon representations of fHbp, bound to $1 \mathrm{~A} 12$ (yellow), JAR5 (blue), $12 \mathrm{C} 1$ (green), and factor $\mathrm{H}$ (red). For clarity, only the Fab variable regions are shown. Top, schematic diagram of the different binding sites on $\mathrm{fHbp}$

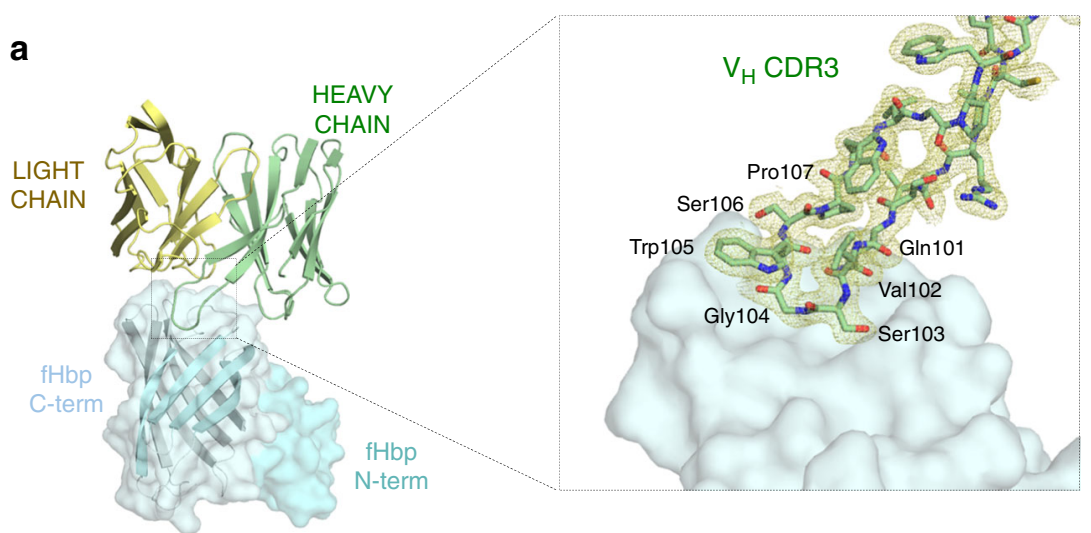

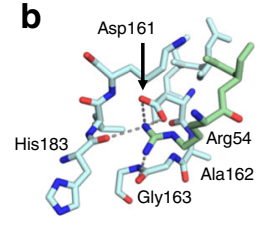

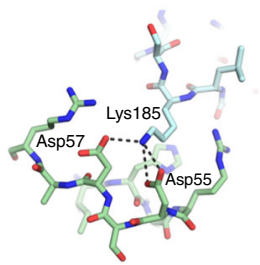

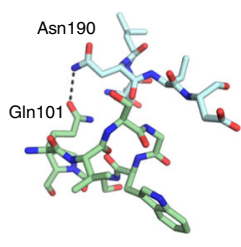

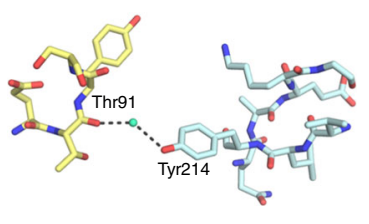

C

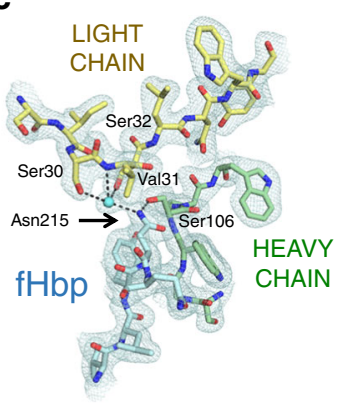

Fig. 4 Intermolecular interactions in the Fab 1A12/fHbp-binding interface. a Left: ribbon representation highlighting the region where the Fab $V_{H}$ CDR3 loop contacts $\mathrm{fHbp}$. The $\mathrm{N}$ - and $\mathrm{C}$-terminal domains of $\mathrm{fHbp}$ are displayed in surface mode in different blue palette colors; the Fab is colored as in Fig. 2. For clarity, the constant regions of the Fab have been omitted. Right: the $\mathrm{V}_{\mathrm{H}}$ CDR3 loop (stick bonds) and its 2Fo-Fc electron density map (yellow mesh) at $1 \sigma$ contour level. Fab constant regions are omitted for clarity. b Noteworthy salt bridges and other polar interactions at the binding interface, involving $V_{H}$ CDR2 and 3. (FHbp: cyan; Fab light chain: yellow; Fab heavy chain: green). c The binding interface centered around fHbp residue Asn215 is shown as sticks. Polar interactions $(\leq 3.3 \AA$ ) established with the heavy and light chains are represented by dashed lines. The cyan sphere represents a water molecule. The blue mesh depicts the 2Fo-Fc electron density map associated with the region displayed, plotted at $1 \sigma$ contour level 

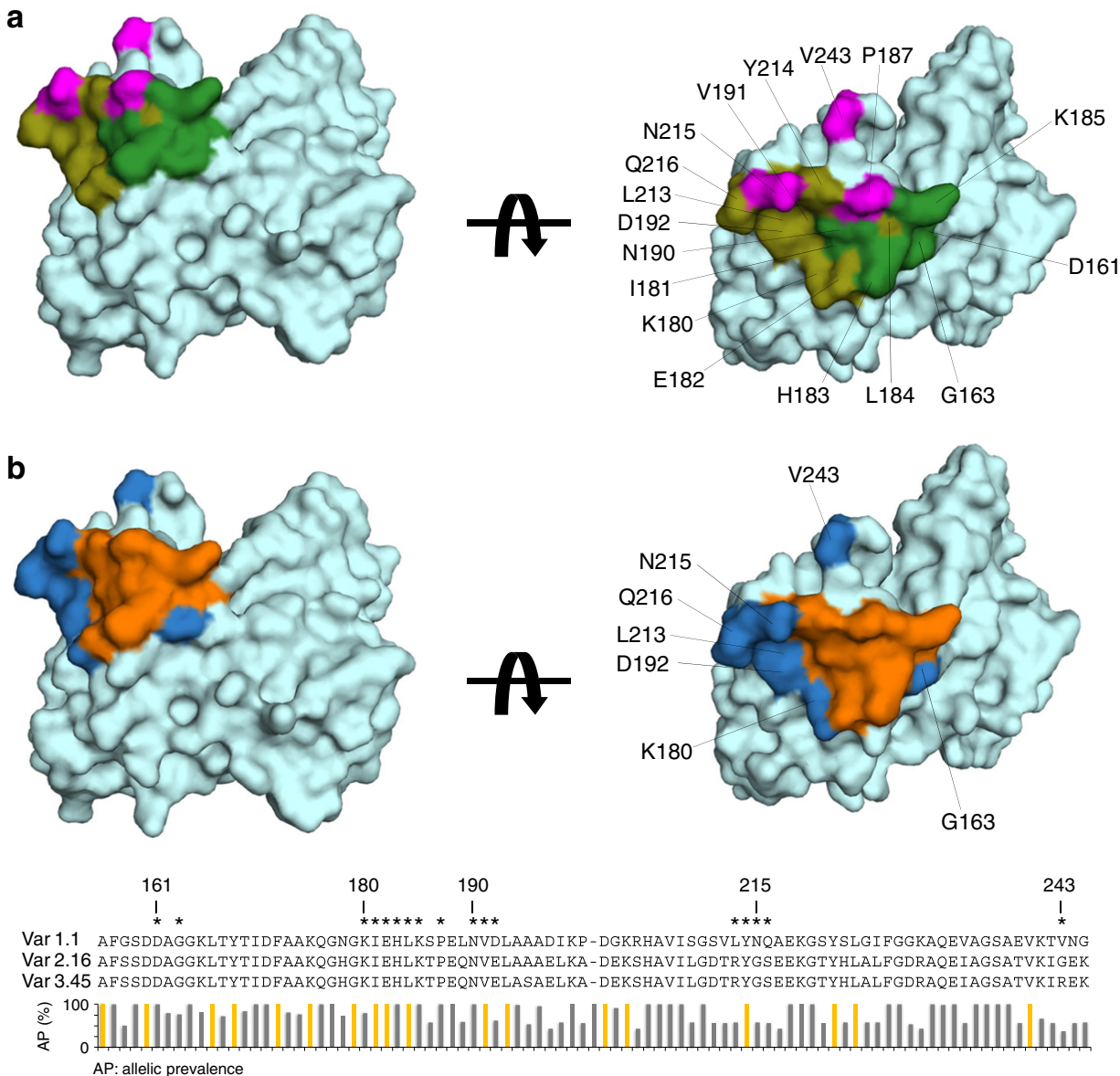

Fig. 5 The 1A12 epitope and its allelic diversity in the fHbp global gene repertoire. a Two views of the 1A12 epitope "footprint" on the surface of fHbp. Residues contacted by the heavy chain are highlighted in green and olive colors for polar and VDW interactions, respectively. The contacts made by the light chain are in magenta. Asn215 establishes polar contacts with both the heavy and light chains. b Allelic diversity in the 1A12 epitope. Upper panel: residues within the $1 \mathrm{~A} 12$ epitope with a degree of conservation $>99 \%$ in all fHbp gene repertoire are colored orange; residues with a prevalence lower than $99 \%$ are shown in dark blue and labeled with their position number. Bottom panel: sequence alignment of fHbp var1.1, 2.16, and 3.45. (The gap at position 200-201 reflects one subvariant of 984 that presents a single-residue insertion (Trp) at this position. Despite the gap, the numbering shown above the alignment corresponds to the numbering used in the main text). The allelic prevalence among $984 \mathrm{fHbp}$ sequences is shown for each position in the $1 \mathrm{~A} 12$ epitope $^{31}$. Orange columns depict sites non-polymorphic in all 984 sequences known. The residues that form the 1A12 epitope are indicated with an asterisk

is a difference in the $\mathrm{V}_{\mathrm{H}} \mathrm{CDR} 3$ loop conformation upon complex formation. Most notably, Gly104 in $\mathrm{V}_{\mathrm{H}}$ CDR3 shifts position by $4 \AA$ A thus avoiding a steric clash with Tyr214 on fHbp (Fig. 7b).

In the complex, Gly104 establishes polar and water-mediated contacts with fHbp residues Asn215 and Gln216 (Fig. 7c). Similarly, the neighboring $\mathrm{V}_{\mathrm{H}}$ CDR3 residues Ser103 and Trp105 also show changes of varying magnitude in their side-chain positions (Fig. 7d), enabling them to make favorable contacts with fHbp. On the other side of the interface, when compared with free $\mathrm{fHbp}^{36}$, it emerges that upon binding most $\mathrm{fHbp}$ residues do not change conformation. One exception is a short loop (fHbp residues 241-246), wherein the alpha carbon of Val243 moves by $3 \AA$ and its side chain undergoes a rotation of $\sim 90^{\circ}$ thereby optimizing contacts with Fab 1A12.

mAb $1 A 12$ recognizes diverse fHbp variants on MenB surface. We sought to understand how the broad cross-reactivity of 1A12 relates to the function of this antibody. We used $1 \mathrm{~A} 12$ as an intact human IgG1 mAb and examined its binding to live bacteria by flow cytometry. We observed that mAb $1 \mathrm{~A} 12$ binds to all three tested MenB strains expressing fHbp from different variant groups: strains H44/76 (fHbp var1.1); M08-0240104 (fHbp var2.16); and M01-0240320 (fHbp var3.45). The var2.16-expressing strain showed the strongest binding, whereas slightly lower levels of binding were observed with the var1.1- and var3.45expressing MenB strains (Fig. 8). The order of binding affinities found by SPR and the degree of binding observed via flow cytometry analysis were different. Assuming that technical differences (between SPR and flow cytometry) do not underlie these observations, we interpret the discrepancy as suggesting that factors other than affinity may affect the overall extent of mAb binding to the live bacterial cells; for example, the antigen density displayed on the bacterial surface. Indeed, the M08-0240104 strain was previously reported to have high expression of $\mathrm{fHbp}$ var2.16, whereas the var1.1 and var3.45 strains were reported to express approximately two- to fourfold lower amounts of $\mathrm{fHbp}$ antigen (Supplementary Table 2) ${ }^{37}$. Nevertheless, these findings confirm the results of SPR analyses in a physiologically more relevant context (live bacterial cells), showing that there is broad cross-recognition by mAb $1 \mathrm{~A} 12$ despite extensive fHbp sequence variability and likely numerous other phenotypic differences existing between diverse meningococcal strains. 

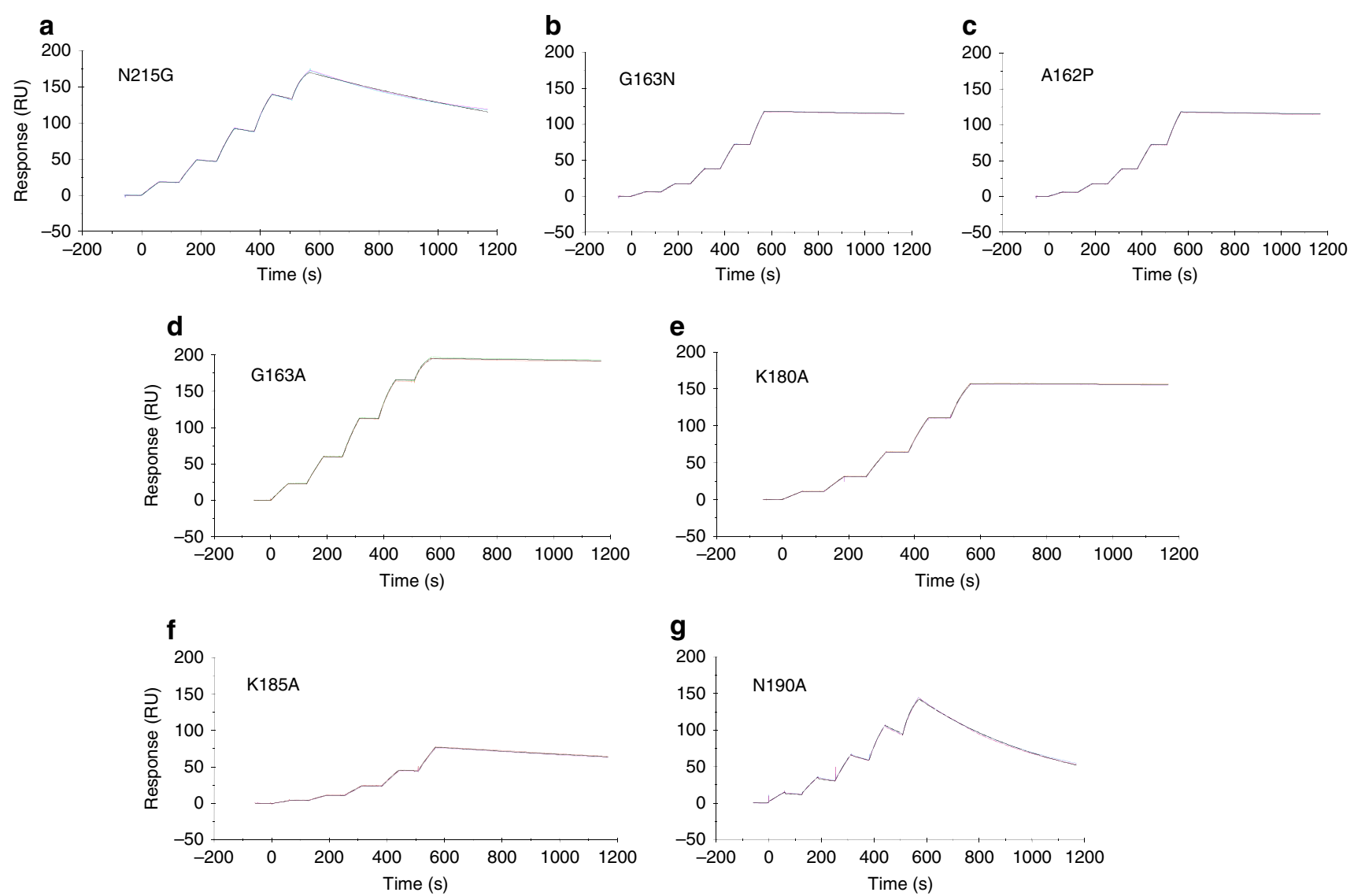

Fig. 6 Impact of fHbp var1.1 site-specific mutations on the binding to mAb 1A12. The effect of the mutations was analyzed by SPR with mAb $1 A 12$ captured on the surface, and the association and the dissociation of diverse mutants (panels a-g) were monitored in duplicate (colored traces). The single-cycle kinetics fitting (Langmuir 1:1 model) is represented as solid black lines in each sensorgram (full details of kinetic analyses are provided in Methods and Table 1)

Bactericidal activity of cross-reactive mAb 1A12. Finally, we investigated whether $\mathrm{mAb} 1 \mathrm{~A} 12$ was bactericidal against MenB strains expressing different $\mathrm{fHbp}$ variants, in an assay measuring complement-dependent killing (using baby rabbit serum as the complement source). We selected this assay to assess the functional activity of the mAb because the serum bactericidal assay (SBA) is the widely accepted functional correlate of protection for Neisseria meningitidis ${ }^{38-40}$. Meningococcal strains H44/76 (expressing fHbp var1.1), M08-0240104 (expressing fHbp var2.16), and M01-0240320 (expressing fHbp var3.45) were incubated with different concentrations of mAb 1A12. Indeed, $\mathrm{mAb} 1 \mathrm{~A} 12$ induced bactericidal activity against all three different strains. The concentrations of $\mathrm{mAb} 1 \mathrm{~A} 12$ required for $\geq 50 \%$ killing of bacteria when measured against strains expressing fHbp var3.45, var2.16, and var1.1 were $0.06,0.49$, and $3.9 \mu \mathrm{g} \mathrm{ml}^{-1}$, respectively. Consequently, $1 \mathrm{~A} 12$ is not only immunologically cross-reactive, but most importantly, it is cross-protective, being able to elicit rabbit complement-dependent bactericidal activity against meningococci in all three of the $\mathrm{fHbp}$ variant groups tested herein.

\section{Discussion}

Vaccines against infectious diseases save an estimated 2-3 million lives each year ${ }^{41}$. Research now targets the development of new vaccines against diseases not yet preventable by current vaccines, and also aims to enhance the breadth of coverage of some current vaccines against highly mutable pathogens. Upon vaccination, protein antigens are recognized by host immune factors (surface- bound B-cell receptors, or soluble antibodies) and elicit protection usually via an antibody-mediated regulatory, or killing, immune response. Therefore, high-resolution structural data of antibody-antigen complexes facilitate an understanding of the molecular bases of immunoprotection, and may consequently aid the development of optimal vaccine immunogens ${ }^{42-44}$. Here we sought structural insights into the human immune recognition of fHbp, a key antigen in the two licensed MenB vaccines.

We determined the crystal structure of $\mathrm{fHbp}$ var1.1 bound to the Fab fragment of a vaccine-elicited human mAb, 1A12. Despite having been generated in a human subject immunized with the fHbp sequence variant 1.1, the mAb $1 \mathrm{~A} 12$ binds with remarkably high affinity (sub-nanomolar $K_{\mathrm{D}}$ ) to representatives from each of the three distinct variant groups of $\mathrm{fHbp}$, and is therefore an antibody of great interest. Our structural and biochemical insights reveal the molecular basis of this crossreactivity. Previous reports of cross-reactive anti-fHbp mAbs were limited to those raised in mice, and mapped their epitopes to $\mathrm{N}$-terminal regions ${ }^{21,23}$, or did not provide any epitope-mapping information ${ }^{30}$. An important implication of our findings is that human immunization with $\mathrm{fHbp}$ var1.1 in the $4 \mathrm{CMenB}$ vaccine may contribute to confer an unexpectedly broad coverage against meningococci expressing fHbp from any of the three known variant groups.

To our knowledge, this is the first report of a vaccine-elicited human Fab bound to a bacterial antigen. One recent report described crystal structures of two human Fabs obtained from memory B cells of healthy donors, and described an unusual mode of recognition of a staphylococcal antigen predominantly 
a

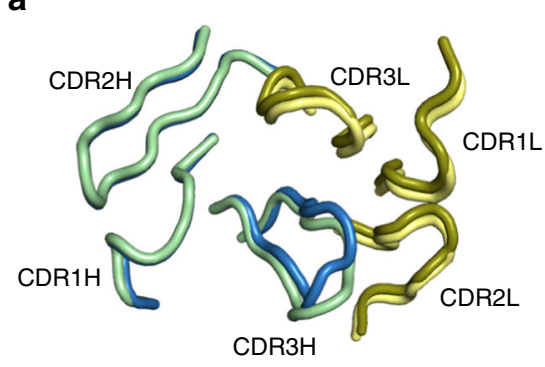

C

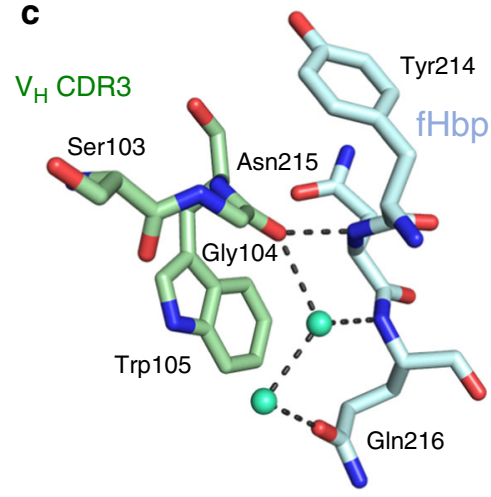

b

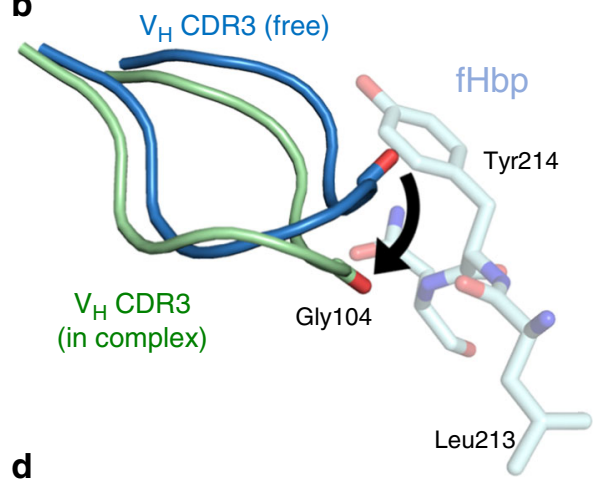

d

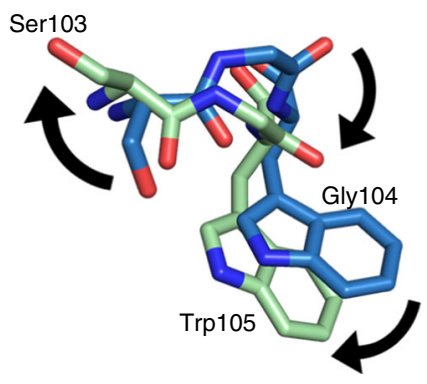

Fig. 7 Conformational changes between bound and free Fab 1A12. a Ribbon diagram showing the light (dark and light yellow) and heavy chains (green and blue) of Fab $1 \mathrm{~A} 12$ both in the liganded (pale colors) and unliganded (dark colors) states. Only CDR3H shows a notable difference. $\mathbf{b} \mathrm{V}_{\mathrm{H}}$ CDR3 loop conformations are represented as cartoons with colors distributed in a similar manner to a; fHbp residue is colored cyan. The movement of Gly104 is indicated. c Detail of the Gly104 region in the bound state. $\mathbf{d}$ Side chains of Ser103 and Trp105 show notably different positions in bound and free forms

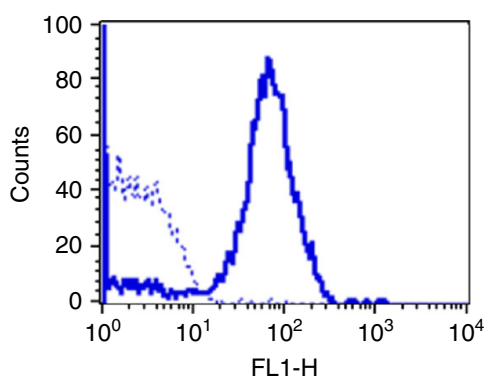

fHbp var1.1

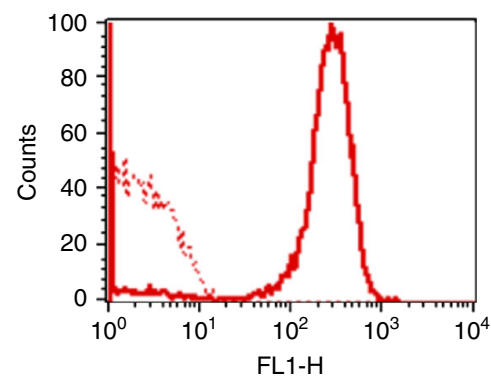

fHbp var2.16

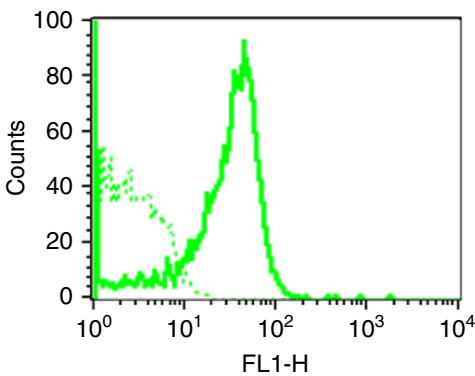

fHbp var3.45

Fig. $8 \mathrm{mAb} 1 \mathrm{~A} 12$ binds meningococci expressing all three fHbp variant groups. Flow cytometry histograms showing the binding of $m A b 1 A 12$ to live serogroup B meningococci H44/76, M08-0240104, and M01-0240320 strains (blue, red and green lines, respectively) when incubated with $10 \mu g \mathrm{ml}^{-1}$ of anti-fHbp mAb. Dotted-line histograms represent negative control, bacteria incubated with PBS and anti-human IgG FITC-conjugated

mediated by $\mathrm{V}_{\mathrm{H}} \mathrm{CDR} 2^{45}$. Here the structure of the $1 \mathrm{~A} 12 / \mathrm{fHbp}$ var1.1 complex shows how the hypervariable $\mathrm{V}_{\mathrm{H}} \mathrm{CDR} 3$ loop interacts with a groove containing several discontinuous residues clustered on a highly solvent-exposed region of the fHbp Cterminal $\beta$ barrel domain. Overall, the recognition of the antigen by Fab $1 \mathrm{~A} 12$ is governed by polar interactions. Numerous $\mathrm{H}-$ bonds, salt bridges, water-dependent contacts, and VDW interactions are widely distributed across the binding interface and contribute collectively to the very strong recognition of fHbp. This cross-reactive conformational epitope presents a unique binding mode that was not previously seen in other crystal structures of fHbp complexed with mAbs raised in mice ${ }^{24,25}$, nor in additional murine mAbs reported to target epitopes on the $\mathrm{N}$ terminal domain of $\mathrm{fHbp}^{21,23}$. Further, comparison of the $1 \mathrm{~A} 12$ epitope and the fH-binding site on $\mathrm{fHbp}^{35}$ reveals two quite distinct interaction areas, and thus provides the structural basis for the lack of inhibition of factor $\mathrm{H}$ binding to $\mathrm{fHbp}$ by human $\mathrm{mAb} 1 \mathrm{~A} 12$, and also confirms that fHbp does not undergo notable conformational changes upon binding to either partner.

Recognition of fHbp by $1 \mathrm{~A} 12$ does not follow the classical "lock and key" concept of antigen-antibody interactions. Rather, although fHbp var1.1 appears relatively rigid, the flexible $\mathrm{V}_{\mathrm{H}}$ CDR3 loop of Fab 1A12 undergoes a notable conformational change, which allows the formation of several favorable interactions with fHbp. The $\mathrm{V}_{\mathrm{H}} \mathrm{CDR} 3$ sequence composition features small residues (Gly and Ser) and a large aromatic residue (Trp), which in itself is not unusual for a $\mathrm{V}_{\mathrm{H}} \mathrm{CDR} 3^{46}$. The availability of both free and antigen-bound states of a Fab ${ }^{47,48}$ allows inspection at high resolution of the functionality in the paratope. In $1 \mathrm{~A} 12$, the presence of Gly and Ser may promote flexibility and allow the 
versatile aromatic residues to mediate numerous interactions with epitope atoms, thus enabling antigen recognition ${ }^{49}$. In short, it appears that the distinct sequence composition of Fab $1 \mathrm{~A} 12$ enables a structural transition in $\mathrm{V}_{\mathrm{H}} \mathrm{CDR} 3$, which translates into an energetically favorable antigen-binding region ideally suited to bind fHbp.

A detailed analysis of the antibody/antigen interface reveals how mAb $1 \mathrm{~A} 12$ can be vastly cross-reactive. In short, of the total $17 \mathrm{fHbp}$ epitope residues that make contact with the Fab, 12 are absolutely conserved, and a further 4 are conserved moderately $(\geq 66 \%)$, in $\mathrm{fHbp}$ var1.1, var2.16, and var3.45. The high conservation of key epitope residues explains the ability of mAb 1A12 to cross-react with the different $\mathrm{fHbp}$ variants (either as purified $\mathrm{fHbp}$ proteins or when expressed on the surface of live meningococci). Moreover, even when a key fHbp epitope residue was mutated to remove its side-chain functionality (N215G), tight binding to mAb $1 \mathrm{~A} 12$ was still observed (sub-nanomolar $K_{\mathrm{D}}$ value). Furthermore, other naturally occurring fHbp substitutions (A162P and G163N) actually increased the strength of mAb binding. These observations suggest that the epitope/paratope interface defined by $1 \mathrm{~A} 12$ can also accommodate at least some known sequence polymorphisms without losing binding functionality.

A vast number of fHbp sequence variants identified from clinical isolates and carrier strains are now known ${ }^{31}$. Therefore, we also analyzed the conservation of the $1 \mathrm{~A} 12$ epitope residues in the 984 subvariants reported to date. We found that several epitope residues are absolutely conserved (5 of 17 residues) throughout the entire fHbp antigenic repertoire, and an additional 5 residues have extremely high $(\geq 99 \%)$ prevalence. Therefore, 10 of 17 epitope residues are at least $99 \%$ conserved in the known antigenic repertoire. Although additional investigations would be needed to demonstrate the full cross-reactivity of mAb $1 \mathrm{~A} 12$ toward the many known subvariants, we envisage a wide recognition of the great majority of $\mathrm{fHbp}$ antigens, with potential to induce bacterial killing either alone or cooperatively with other mAbs against fHbp or in synergy with antibodies against alternative MenB surface antigens. The observation that antibodies recognizing ordered conformational epitopes are less sensitive to antigen sequence diversity than those antibodies targeting disordered epitopes ${ }^{33}$ further underscores the likelihood that $\mathrm{mAb} 1 \mathrm{~A} 12$ may react with most $\mathrm{fHbp}$ variants.

We found that mAb $1 \mathrm{~A} 12$ bound tightly to all three variants of $\mathrm{fHbp}$ when tested in biochemical assays (SPR), and live cell-based binding assays (flow cytometry). Interestingly however, we found that the binding affinities determined with the soluble recombinant proteins did not correlate closely with the amount of binding to whole bacteria as measured by flow cytometry (Table 2). Using the selected reaction monitoring mass spectrometry approach, the surface concentration of $\mathrm{fHbp}$ in these strains was previously determined to be $\sim 4000,9000$, and 1000 molecules per cell for variants var1.1, var2.16, and var3.45, respectively ${ }^{37}$. Given that mAb $1 \mathrm{~A} 12$ showed very high affinity for each $\mathrm{fHbp}$ subvariant, it is likely that the higher antigen density on var2.16 underlies the higher fluorescence response measured here for the var2.16 strain.

Importantly, mAb $1 \mathrm{~A} 12$ not only recognized the three $\mathrm{fHbp}$ variants on the surface of live meningococci, it was also able to activate the complement cascade and induce bacterial killing against MenB strains expressing fHbp var1, var2, or var3 antigens, as demonstrated in bactericidal assays, here using baby rabbit serum as the complement source. That is, mAb $1 \mathrm{~A} 12$ is cross-protective as well as being cross-reactive. Some antimeningococcal mAbs have bactericidal activity only when combined with other mAbs targeting different epitopes or even different antigens on the same bacterial cell. In contrast, mAb $1 \mathrm{~A} 12$ is able to induce the killing alone (with higher efficiencies for var2 and var3 strains), presumably through highly efficient activation of the classical pathway of the complement system, which highlights the benefit of immunologically targeting this epitope on fHbp. Somewhat counter-intuitively, we observed that the killing activity was strongest against the M01-0240320 (fHbp var3.45) strain, where the surface density of $\mathrm{fHbp}$ is the lowest. It is possible either that the M01-0240320 strain is inherently more susceptible to killing, or that the particular fHbp var3.45 antigen density on this strain was sterically or geometrically more efficient for mAb 1A12-dependent activation of the complement pathway, or both. While the susceptibility to complement-mediated killing (using polyclonal anti-fHbp sera in the SBA assay) has been shown to correlate with the absolute amount of $\mathrm{fHbp}$ protein expressed by each meningococcal strain $^{37}$, some additional strain-specific differences in the intrinsic susceptibility to killing by unique mAbs are likely determined by other factors, such as the expression of virulence molecules that bind host complement regulators ${ }^{50}$.

The most efficient complement-dependent immune response against a specific surface antigen may result from the activity of two or more different mAbs engaging the same antigen simultaneously ${ }^{25}$. In general, it is not the action of only one $\mathrm{mAb}$ but the combination of different $\mathrm{mAbs}$ in a polyclonal response that are directed against alternative noncompeting epitopes that will act cooperatively to maximize the efficiency of the immune response ${ }^{51}$. Therefore, the crossprotective human $\mathrm{mAb} 1 \mathrm{~A} 12$ characterized here appears to be a potentially key player in such a multivalent bactericidal response. The extent to which such a cross-reactive mAb could contribute to meningococcal killing in vivo in a vaccinated individual may depend on its IgG subclass and will of course also depend on the absolute quantity in which the $\mathrm{mAb}$ is present $^{52}$. While it was beyond the scope of this study to determine the serum concentrations of individual mAbs, recently published proteomic approaches combined with nextgeneration sequencing have demonstrated that a molecular deconvolution of the immune response can be performed ${ }^{53}$, and this might form the basis of future studies to further explore the response to meningococcal vaccines such as 4CMenB.

In summary, we present here the crystal structures of an fHbpspecific human Fab in free and antigen-bound states, elicited by vaccination. We define a molecular signature that allows a vaccine-elicited human $\mathrm{mAb}$ to cross-react with the three different variants of fHbp and importantly, to induce complementdependent killing responses against MenB strains harboring $\mathrm{fHbp}$ antigens from variants 1,2 , or 3 . The existence of this crossprotective epitope on $\mathrm{fHb}$ var1.1 suggests that the broad efficacy demonstrated by the $4 \mathrm{CMenB}$ vaccination in the United Kingdom $^{10,11}$ could result from a multi-factorial effect, where antigens carrying cross-protective epitopes play key synergistic roles. Moreover, such detailed structural studies could be exploited for the design of vaccines with an immunofocusing approach toward cross-protective epitopes, aiming to further enhance the existing breadth of protection. More broadly, it is noteworthy that several current vaccines against bacterial pathogens are essentially based on surface-exposed polysaccharides that make up the outermost layer of the bacterial surface. However, when capsular polysaccharides are unsuitable vaccine candidates, or when polysaccharide serotypes are too numerous and variable, alternative reverse and structural vaccinology approaches may permit the identification and design of protein-based epitope-focused vaccine candidates. In this light, our studies provide an exploratory human vaccination model enabling the identification of broadly protective epitopes that could be expanded to the design of ideal saccharide-independent cross-protective bacterial targets. 


\section{Methods}

Human samples. Human peripheral blood mononuclear cells were collected from three vaccinated subjects 8 days after the second dose of a multicomponent serogroup B meningococcal vaccine containing recombinant $\mathrm{fHbp}$ variant 1.1. Plasmablasts were processed individually (not pooled), were isolated as single cells, and were used as the source from which genes of heavy and light chain variable regions were amplified separately and then combined by overlap extension PCR, in order to obtain Fab region sequences. Recombinant Fabs were then produced in E. coli (see Protein expression description below) and were screened for antigen specificity through ELISA assays involving detection of fHbp-bound Fabs by Fab-specific goat anti-human IgG conjugated to alkaline phosphatase (Sigma; 1:5000 in phosphatebuffered saline (PBS)-Tween 20-bovine serum albumin (BSA) ${ }^{16}$. The samples were obtained from a clinical trial conducted in Krakow, Poland, in a study sponsored by Novartis Vaccines \& Diagnostics, now part of the GSK group of companies. The clinical trial protocol was approved by the Bioethics Committee of the District Medical Doctors' Chamber in Krakow and the study was conducted in accordance with the Declaration of Helsinki. Written informed consent was obtained from each of the subjects.

Protein expression. All genes for the preparation of the fHbp subvariants and point mutants used in this study were cloned and amplified using DH5 $\alpha$ and MultiShot $^{\mathrm{TM}}$ StripWell Mach $1^{\mathrm{TM}}$ T1 Phage-Resistant Chemically Competent $E$. coli cells (Invitrogen), expressed from pET vectors (Novagen) induced by Isopropyl- $\beta$-D-thiogalactoside in E. coli strain BL21 (DE3) (New England Biolabs) and purified via C-terminal 6-His tags using $\mathrm{Ni}^{2+}$-affinity chromatography columns (His-Trap HP, $1 \mathrm{ml}$, GE Healthcare) and buffer solutions recommended by the manufacturer, controlled by an ÄKTA Purifier liquid chromatography system (GE Healthcare). PCR primers used to generate fHbp point mutants are listed (Supplementary Table 3). Full-length factor $\mathrm{H}$ was purchased from Calbiochem. For the expression of mAb 1A12, the variable regions of the heavy and light chains of 1A12 were codon-optimized (Supplementary Table 4) for expression in mammalian cells and synthesized by GeneArt (Thermo Fisher). Synthetic DNA sequences were digested with EcoRI (New England Biolabs) and cloned into the human pRS5a expression vectors encoding the Ig 1 and Igk backbone, under the control of the cytomegalovirus promoter and in frame with a leader sequence for secretion derived from human immunoglobulins (Novartis-NIBR). The recombinant antibody was transiently expressed in Expi293 cells by transfecting the cells with equivalent amounts of both plasmids with the use of the Expi293 expression system (Thermo Fisher). Three and six days after transfection, cells were harvested, centrifuged for $10 \mathrm{~min}$ at $350 \times g$, and filtered through a $0.2 \mu \mathrm{m}$ filter to remove cellular debris. Recombinant antibody was purified from the tissue culture expression medium with Protein G Sepharose 4 Fast Flow (GE Healthcare), following the manufacturer's protocol. A PD-10 Desalting Column (GE Healthcare) was used for buffer exchange and the antibody was eluted in PBS pH 7.4. $1 \mathrm{~A} 12 \mathrm{IgG}$ concentration was determined in a NanoDrop spectrophotometer (Thermo Scientific) and its purity was assessed by SDS-PAGE on a $4-12 \%$ Bis-Tris Gel and Problue Safe Stain (Giotto Biotech).

The recombinant plasmid for human Fab 1A12 and the expression in E. coli (New England Biolabs) have been previously described ${ }^{16}$. The bacteria were suspended in $50 \mathrm{mM} \mathrm{NaH} \mathrm{PO}_{4}, 500 \mathrm{mM} \mathrm{NaCl}, 20 \mathrm{mM}$ imidazole, pH 7.0, and lysed using chicken egg white lysozyme, DNase, and RNase (Sigma; $0.1 \mathrm{mg} \mathrm{ml}^{-1}$ each), and three freeze/thaw cycles. The clarified lysate was applied to a HiTrap Chelating HP ( $5 \mathrm{ml}$; GE Healthcare) column and the bound protein was eluted with an imidazole gradient from 20 to $250 \mathrm{mM}$. The Fab was further purified by cation exchange chromatography (HiTrap SP HP $5 \mathrm{ml}$; GE Healthcare) using 20 $\mathrm{mM}$ sodium acetate buffer, $\mathrm{pH} 5.5$, and elution with a $\mathrm{NaCl}$ gradient from 0.02 to 1.0 M. Fractions containing the Fab were dialyzed against $20 \mathrm{mM}$ Tris- $\mathrm{HCl}, 20 \mathrm{mM}$ $\mathrm{NaCl}, \mathrm{pH} 7.0$ for crystallization trials.

For formation of the complex, fHbp var1.1 was expressed and purified as described above. Fab-expressing E. coli cells were first sonicated in ice-cold $10 \mathrm{mM}$ HEPES ( $\mathrm{pH} 7.4$ ) and $150 \mathrm{mM} \mathrm{NaCl}$, and centrifuged at $9500 \times g$ for $30 \mathrm{~min}$. The supernatant was then filtered and loaded on a $\mathrm{Ni}^{2+}$ Sepharose 6 Fast Flow column (GE Healthcare) pre-saturated with recombinant fHbp var1.1. The bound protein was eluted with $10 \mathrm{mM}$ HEPES (pH 7.4), $150 \mathrm{mM} \mathrm{NaCl}$, and $300 \mathrm{mM}$ imidazole. Next, the protein was subjected to three cycles of concentration and dilution with $10 \mathrm{mM}$ HEPES ( $\mathrm{pH} 7.4$ ) and $150 \mathrm{mM} \mathrm{NaCl}$ using an Amicon concentrator (Millipore) with a $30 \mathrm{kDa}$ cutoff. The complex was then recovered for crystallization assays.

Surface plasmon resonance. All interaction experiments were performed using a BIAcore T200 instrument (GE Healthcare), equilibrated at $25^{\circ} \mathrm{C}$. First, the mAb $1 \mathrm{~A} 12$ was captured to a density of $\sim 540$ resonance units on the surface of a CM5 sensor chip previously coated with covalently immobilized monoclonal mouse anti-human $\operatorname{IgG}(\mathrm{Fc})$ antibody (GE Healthcare). In order to subtract the background signal for kinetic analysis, we prepared a control reference channel in a similar way but in the absence of the mAb. A series of concentrations of the different fHbp variants (wild type or mutants) were then injected in 0.01 M HEPES ( $\mathrm{pH}$ 7.4), $0.15 \mathrm{M} \mathrm{NaCl}$ and $3 \mathrm{mM}$ EDTA. To minimize nonspecific interactions, the running buffer was also supplemented with $0.005 \% \mathrm{v} / \mathrm{v}$ surfactant P20 and $0.1 \%$ (w/v) BSA. All kinetic parameters were calculated using standard single-cycle kinetics fitting and a Langmuir 1:1 binding model (BIAcore T200 evaluation software).

Crystallization and diffraction data. The purified Fab $\left(7.1 \mathrm{mg} \mathrm{ml}^{-1}\right)$ was screened against 96 crystallization reagents (Index Screen, Hampton Research). Crystals were observed in multiple conditions; after 4 days, the largest crystals were found in $60 \%$ Tacsimate, $\mathrm{pH}$ 7.0. Optimization of the crystallization conditions was achieved in 57.5\% Tacsimate (Hampton Research) at pH 6.0. Crystals were soaked in $15 \%$ PEG 3350 and $12 \%$ Tacsimate as cryoprotectant and cryo-cooled in liquid nitrogen The crystals were tested for diffraction at beamline 5.0.1 at the Advanced Light Source, Lawrence Berkeley National Laboratory and several data sets were collected on an ADSC Q315R detector (Table 2). Data were integrated and scaled with iMosflm ${ }^{54}$. The structure of the Fab was solved using Phaser ${ }^{55}$ with a homology model constructed from the $1 \mathrm{~A} 12$ sequence and the coordinates of the human anti HIV 21c Fab (PDB ID 3LMJ) using Swiss-Model ${ }^{56}$.

The Fab 1A12-fHbp complex at $10 \mathrm{mg} \mathrm{ml}^{-1}$ was screened with a matrix of 800 crystallization conditions using a Crystal Gryphon robot (Art Robbins Instruments). Long plate-needle-looking crystals were found at days 7-10 with $17 \%$ PEG 3350 and $0.2 \mathrm{M}$ sodium malonate ( $\mathrm{pH} 4.0$ ). Optimization of the original conditions was performed by changing the concentrations of the different components in the initial crystallization mixture. Changes in the $\mathrm{pH}$ did not yield any improvement. Crystals were soaked in the original mother liquor supplemented with $10 \%$ ethylene glycol as cryoprotectant and prior to cryo-cooling in liquid nitrogen. Diffraction of the crystals was tested at beamline ID29 of the European Synchrotron Radiation Facility and several full data sets were collected on a Pilatus 6M detector. Diffraction data sets were indexed, integrated, and scaled with $\mathrm{XDS}^{57}$ and Aimless ${ }^{58}$, via the CCP4 suite ${ }^{59}$. The structure of the complex was solved by molecular replacement with Phaser ${ }^{55}$ using as model templates for $\mathrm{fHbp}$, light chain, and heavy chains the coordinates deposited in the protein data bank under the codes 2 YPV, 4YPG, and 4JQI, respectively. The CDR3 loop of the heavy chain was omitted in the template.

Structure refinement. Initial molecular replacement solutions were subjected to subsequent cycles of manual building in $\mathrm{Coot}^{60}$ and refinement with Phenix. refine $^{61}$. The buried surface areas and atomic interactions/contacts, and the root mean square displacements, were calculated with PISA ${ }^{62}$ and Superpose ${ }^{63}$, respectively. All the structural figures were created with $\mathrm{PyMOL}^{64}$.

Flow cytometry analysis. The ability of mAb $1 \mathrm{~A} 12$ to bind the antigen exposed on the surface of $N$. meningitidis bacteria, expressing different fHbp variants, was determined using a FACScan flow cytometer. Bacteria grown until early log phase (OD600 of $\sim 0.25$ ) were incubated with $\mathrm{mAb}$ at the concentration of $10 \mu \mathrm{g} \mathrm{ml}^{-1}$. Antibody binding was detected using a goat anti-Human IgG conjugated to fluorescein isothiocyanate (Jackson Immuno Research, catalog number 109-096088 ) at a 1:100 dilution. Bacteria plus PBS-1\% BSA and secondary antibody were used as negative control.

Bactericidal assay. Bacteria grown in Mueller Hinton broth supplemented with $0.25 \%$ glucose until early log phase (OD600 of $\sim 0.25$ ) were diluted in Dulbecco's PBS containing $1 \%$ BSA and $0.1 \%$ glucose at the working dilution of $10^{4}-10^{5}$ colony forming units (CFU) per $\mathrm{ml}$ and incubated with serial twofold dilutions of test $\mathrm{mAb}$ starting from a concentration of $31.25 \mu \mathrm{g} \mathrm{m} l^{-1}$ (corresponding to $1 / 16$ dilution in the reaction mixture in the well). Bactericidal titers were defined as the reciprocal $\mathrm{mAb}$ dilution resulting in $50 \%$ decrease in CFU per $\mathrm{ml}$ after a 60 -min incubation of bacteria with the reaction mixture compared to the control CFU per $\mathrm{ml}$ at time zero. Pooled baby rabbit serum (Cedarlane) was used as a complement source.

Data availability. Structure factors and atomic coordinates have been deposited in the Protein Data Bank for the Fab 1A12 (ID 5UR8) and Fab 1A12-fHbp var1.1 complex (ID 5O14). Other data are available from the corresponding authors upon reasonable request.

Received: 22 June 2017 Accepted: 3 January 2018

Published online: 06 February 2018

\section{References}

1. European Centre for Disease Prevention and Control. Annual Epidemiological Report on Communicable Diseases in Europe 2010. (Stockholm, ECDC, 2010).

2. Sadarangani, M. \& Pollard, A. J. Can we control all-cause meningococcal disease in Europe? Clin. Microbiol. Infect. 22 Suppl 5, S103-S112 (2016). 
3. Masignani, V. et al. Vaccination against Neisseria meningitidis using three variants of the lipoprotein GNA1870. J. Exp. Med. 197, 789-799 (2003).

4. Fletcher, L. D. et al. Vaccine potential of the Neisseria meningitidis 2086 lipoprotein. Infect. Immun. 72, 2088-2100 (2004).

5. Jiang, H. Q. et al. Broad vaccine coverage predicted for a bivalent recombinant factor $\mathrm{H}$ binding protein based vaccine to prevent serogroup $\mathrm{B}$ meningococcal disease. Vaccine 28, 6086-6093 (2010).

6. Koeberling, O. et al. Immunogenicity of a meningococcal native outer membrane vesicle vaccine with attenuated endotoxin and over-expressed factor $\mathrm{H}$ binding protein in infant rhesus monkeys. Vaccine 29, 4728-4734 (2011).

7. Findlow, J. et al. Multicenter, open-label, randomized phase II controlled trial of an investigational recombinant Meningococcal serogroup B vaccine with and without outer membrane vesicles, administered in infancy. Clin. Infect. Dis. 51, 1127-1137 (2010).

8. Marshall, H. S. et al. Safety and immunogenicity of a meningococcal B bivalent rLP2086 vaccine in healthy toddlers aged 18-36 months: a phase 1 randomized-controlled clinical trial. Pediatr. Infect. Dis. J. 31, 1061-1068 (2012).

9. Marshall, H. S. et al. A phase 2 open-label safety and immunogenicity study of a meningococcal B bivalent rLP2086 vaccine in healthy adults. Vaccine 31, 1569-1575 (2013).

10. Basta, N. E. \& Christensen, H. 4CMenB vaccine effectiveness: reasons for optimism. Lancet 388, 2719-2721 (2016).

11. Parikh, S. R. et al. Effectiveness and impact of a reduced infant schedule of 4CMenB vaccine against group B meningococcal disease in England: a national observational cohort study. Lancet 388, 2775-2782 (2016).

12. Madico, G. et al. The meningococcal vaccine candidate GNA1870 binds the complement regulatory protein factor $\mathrm{H}$ and enhances serum resistance. J. Immunol. 177, 501-510 (2006).

13. Costa, I., Pajon, R. \& Granoff, D. M. Human factor H (FH) impairs protective meningococcal anti-FHbp antibody responses and the antibodies enhance $\mathrm{FH}$ binding. MBio 5, e01625-14 (2014).

14. Granoff, D. M. et al. Enhanced protective antibody to a mutant meningococcal factor H-binding protein with low-factor $\mathrm{H}$ binding. JCI Insight 1, e88907 (2016).

15. Granoff, D. M. et al. Binding of complement factor $\mathrm{H}(\mathrm{FH})$ decreases protective anti-FH binding protein antibody responses of infant rhesus macaques immunized with a meningococcal serogroup B vaccine. J. Infect. Dis. 212, 784-792 (2015)

16. Beernink, P. T., Giuntini, S., Costa, I., Lucas, A. H. \& Granoff, D. M. Functional analysis of the human antibody response to meningococcal factor $\mathrm{H}$ binding protein. MBio 6, e00842 (2015).

17. Giuliani, M. M. et al. The region comprising amino acids 100 to 255 of Neisseria meningitidis lipoprotein GNA 1870 elicits bactericidal antibodies. Infect. Immun. 73, 1151-1160 (2005)

18. Scarselli, M. et al. Epitope mapping of a bactericidal monoclonal antibody against the factor $\mathrm{H}$ binding protein of Neisseria meningitidis. J. Mol. Biol. 386, 97-108 (2009).

19. Beernink, P. T. et al. Fine antigenic specificity and cooperative bactericidal activity of monoclonal antibodies directed at the meningococcal vaccine candidate factor $\mathrm{h}$-binding protein. Infect Immun. 76, 4232-4240 (2008).

20. Beernink, P. T., LoPasso, C., Angiolillo, A., Felici, F. \& Granoff, D. A region of the $\mathrm{N}$-terminal domain of meningococcal factor $\mathrm{H}$-binding protein that elicits bactericidal antibody across antigenic variant groups. Mol. Immunol. 46, 1647-1653 (2009).

21. Faleri, A. et al. Two cross-reactive monoclonal antibodies recognize overlapping epitopes on Neisseria meningitidis factor $\mathrm{H}$ binding protein but have different functional properties. FASEB J. 28, 1644-1653 (2014).

22. Mascioni, A. et al. Structural basis for the immunogenic properties of the meningococcal vaccine candidate LP2086. J. Biol. Chem. 284, 8738-8746 (2009).

23. Vu, D. M., Pajon, R., Reason, D. C. \& Granoff, D. M. A broadly cross-reactive monoclonal antibody against an epitope on the $\mathrm{N}$-terminus of meningococcal fHbp. Sci. Rep. 2, 341 (2012).

24. Malito, E. et al. Defining a protective epitope on factor $\mathrm{H}$ binding protein, a key meningococcal virulence factor and vaccine antigen. Proc. Natl Acad. Sci. USA 110, 3304-3309 (2013).

25. Malito, E. et al. Neisseria meningitidis factor H-binding protein bound to monoclonal antibody JAR5: implications for antibody synergy. Biochem. J. 473, 4699-4713 (2016).

26. Klontz, E. H. \& Sundberg, E. J. Getting oriented with antibodies. Biochem. J. 474, 517-519 (2017).

27. Derrick, J. P., Maiden, M. C. \& Feavers, I. M. Crystal structure of an Fab fragment in complex with a meningococcal serosubtype antigen and a protein G domain. J. Mol. Biol. 293, 81-91 (1999).
28. Oomen, C. J. et al. Crystal structure of an anti-meningococcal subtype P1.4 PorA antibody provides basis for peptide-vaccine design. J. Mol. Biol. 351, 1070-1080 (2005)

29. van den Elsen, J. M. et al. Bactericidal antibody recognition of a PorA epitope of Neisseria meningitidis: crystal structure of a Fab fragment in complex with a fluorescein-conjugated peptide. Proteins 29, 113-125 (1997).

30. McNeil, L. K. et al. Detection of LP2086 on the cell surface of Neisseria meningitidis and its accessibility in the presence of serogroup B capsular polysaccharide. Vaccine 27, 3417-3421 (2009).

31. Jolley, K. A. \& Maiden, M. C. BIGSdb: scalable analysis of bacterial genome variation at the population level. BMC Bioinformatics 11, 595 (2010).

32. Diederichs, K. Crystallographic data and model quality. Methods Mol. Biol. 1320, 147-173 (2016).

33. MacRaild, C. A., Richards, J. S., Anders, R. F. \& Norton, R. S. Antibody recognition of disordered antigens. Structure 24, 148-157 (2016).

34. Rubinstein, N. D. et al. Computational characterization of B-cell epitopes. Mol. Immunol. 45, 3477-3489 (2008).

35. Schneider, M. C. et al. Neisseria meningitidis recruits factor $\mathrm{H}$ using protein mimicry of host carbohydrates. Nature 458, 890-893 (2009).

36. Cendron, L., Veggi, D., Girardi, E. \& Zanotti, G. Structure of the uncomplexed Neisseria meningitidis factor H-binding protein $\mathrm{fHbp}$ (rLP2086). Acta Crystallogr. F Struct. Biol. Cryst. Commun. 67, 531-535 (2011).

37. Biagini, $\mathrm{M}$. et al. Expression of factor $\mathrm{H}$ binding protein in meningococcal strains can vary at least 15 -fold and is genetically determined. Proc. Natl Acad. Sci. USA 113, 2714-2719 (2016).

38. Borrow, R., Andrews, N., Goldblatt, D. \& Miller, E. Serological basis for use of meningococcal serogroup C conjugate vaccines in the United Kingdom: reevaluation of correlates of protection. Infect. Immun. 69, 1568-1573 (2001).

39. Frasch, C. E., Borrow, R. \& Donnelly, J. Bactericidal antibody is the immunologic surrogate of protection against meningococcal disease. Vaccine 27 Suppl 2, B112-B116 (2009).

40. Poolman, J. T. \& Richmond, P. Multivalent meningococcal serogroup B vaccines: challenges in predicting protection and measuring effectiveness. Expert Rev. Vaccines 14, 1277-1287 (2015).

41. Delany, I., Rappuoli, R. \& De Gregorio, E. Vaccines for the 21st century. EMBO Mol. Med. 6, 708-720 (2014).

42. Liljeroos, L., Malito, E., Ferlenghi, I. \& Bottomley, M. J. Structural and computational biology in the design of immunogenic vaccine antigens. $J$. Immunol. Res. 2015, 156241 (2015).

43. Malito, E., Carfi, A. \& Bottomley, M. J. Protein crystallography in vaccine research and development. Int J. Mol. Sci. 16, 13106-13140 (2015).

44. Rappuoli, R., Bottomley, M. J., D'Oro, U., Finco, O. \& De Gregorio, E. Reverse vaccinology 2.0: human immunology instructs vaccine antigen design. J. Exp. Med. 213, 469-481 (2016)

45. Yeung, Y. A. et al. Germline-encoded neutralization of a Staphylococcus aureus virulence factor by the human antibody repertoire. Nat. Commun. 7, 13376 (2016)

46. Ramaraj, T., Angel, T., Dratz, E. A., Jesaitis, A. J. \& Mumey, B. Antigenantibody interface properties: composition, residue interactions, and features of 53 non-redundant structures. Biochim. Biophys. Acta 1824, 520-532 (2012).

47. Li, Y., Li, H., Smith-Gill, S. J. \& Mariuzza, R. A. Three-dimensional structures of the free and antigen-bound Fab from monoclonal antilysozyme antibody HyHEL-63(,). Biochemistry 39, 6296-6309 (2000).

48. Rini, J. M., Schulze-Gahmen, U. \& Wilson, I. A. Structural evidence for induced fit as a mechanism for antibody-antigen recognition. Science $\mathbf{2 5 5}$, 959-965 (1992).

49. Birtalan, S. et al. The intrinsic contributions of tyrosine, serine, glycine and arginine to the affinity and specificity of antibodies. J. Mol. Biol. 377, 1518-1528 (2008).

50. Rossi, R., Beernink, P. T., Giuntini, S. \& Granoff, D. M. Susceptibility of meningococcal strains responsible for two serogroup B outbreaks on U.S. University Campuses to serum bactericidal activity elicited by the MenB-4C vaccine. Clin. Vaccine Immunol. 22, 1227-1234 (2015).

51. Sorman, A., Zhang, L., Ding, Z. \& Heyman, B. How antibodies use complement to regulate antibody responses. Mol. Immunol. 61, 79-88 (2014).

52. Giuntini, S. et al. Human IgG1, IgG3, and IgG3 hinge-truncated mutants show different protection capabilities against meningococci depending on the target antigen and epitope specificity. Clin. Vaccine Immunol. 23, 698-706 (2016).

53. Wine, Y. et al. Molecular deconvolution of the monoclonal antibodies that comprise the polyclonal serum response. Proc. Natl Acad. Sci. USA 110, 2993-2998 (2013)

54. Battye, T. G., Kontogiannis, L., Johnson, O., Powell, H. R. \& Leslie, A. G. iMOSFLM: a new graphical interface for diffraction-image processing with MOSFLM. Acta Crystallogr. D. Biol. Crystallogr. 67, 271-281 (2011).

55. McCoy, A. J. et al. Phaser crystallographic software. J. Appl. Crystallogr. 40, 658-674 (2007). 
56. Biasini, M. et al. SWISS-MODEL: modelling protein tertiary and quaternary structure using evolutionary information. Nucleic Acids Res. 42, W252-W258 (2014).

57. Kabsch, W. Xds. Acta Crystallogr D. Biol. Crystallogr. 66, 125-132 (2010).

58. Evans, P. R. \& Murshudov, G. N. How good are my data and what is the resolution? Acta Crystallogr. D. Biol. Crystallogr. 69, 1204-1214 (2013).

59. Collaborative Computational Project, N. The CCP4 suite: programs for protein crystallography. Acta Crystallogr. D. Biol. Crystallogr. 50, 760-763 (1994).

60. Emsley, P., Lohkamp, B., Scott, W. G. \& Cowtan, K. Features and development of Coot. Acta Crystallogr. D. Biol. Crystallogr. 66, 486-501 (2010).

61. Adams, P. D. et al. PHENIX: a comprehensive Python-based system for macromolecular structure solution. Acta Crystallogr. D. Biol. Crystallogr. 66, 213-221 (2010).

62. Krissinel, E. \& Henrick, K. Inference of macromolecular assemblies from crystalline state. J. Mol. Biol. 372, 774-797 (2007).

63. Krissinel E. and Henrick K. Secondary-structure matching (SSM), a new tool for fast protein structure alignment in three dimension. Acta Crystallogr. D. Biol. Crystallogr. D60, 2256-2268 (2004).

64. The PyMOL Molecular Graphics System, Version 1.8. (Schrodinger, LLC, 2015)

\section{Acknowledgements}

We wish to thank the clinical study participants. We thank Drs Sumana Chandramouli, Oretta Finco, Paola Lo Surdo, Domenico Maione, Enrico Malito, and Vega Masignani at GSK, Siena, for useful discussions during this project. We thank Cecilia Brettoni for provision of fHbp var1.1 mutant clones, and Sara Marchi for fHbp proteins. We thank Elias Tsadik, Heather Stefek, and Emily Arnold (UCSF Benioff Children's Hospital Oakland) for technical assistance and staff at beam lines ID29 of the European Synchrotron Radiation Facility (ESRF, France) and 5.0.1 at the Advanced Light Source, Lawrence Berkeley National Laboratory for their assistance in collecting X-ray diffraction data. N. meningitidis strains used in this work were kindly provided: $\mathrm{H} 44 / 76$ by Dominique A. Caugant (Norwegian Institute of Public Health, Oslo, Norway); M080240104 and M01-0240320 by Ray Borrow (Health Protection Agency, Manchester, UK). This study was sponsored by GSK Vaccines srl (Siena, Italy). J.L.-S. was funded by a Marie Skłodowska-Curie Actions IF grant via the European Commission Horizon 2020 program (grant 659615). During this project, F.B. held a PhD Fellowship registered at the University of Florence, Italy. The work was supported in part by extramural research grants (R01 AI099125 and R01 AI114701) from the National Institute of Allergy and Infectious Diseases, National Institutes of Health, to P.T.B. The Advanced Light Source is supported by the Director, Office of Science, Office of Basic Energy Sciences, of the U.S.
Department of Energy under Contract No. DE-AC02-05CH11231. Bexsero is a trademark of the GSK group of companies. Trumenba is a trademark of Pfizer.

\section{Author contributions}

Conceived and designed the experiments: J.L.-S.; P.T.B.; A.H.L.; and M.J.B. Performed the experiments: J.L.-S.; P.T.B.; F.B.; L.S.; and E.F. Analyzed the data: J.L.-S.; P.T.B.; M.J.B.; and M.P. Wrote the paper: J.L.-S.; P.T.B., and M.J.B. All authors reviewed and approved the manuscript.

\section{Additional information}

Supplementary Information accompanies this paper at https://doi.org/10.1038/s41467018-02827-7.

Competing interests: J.L.-S., L.S., E.F., M.P., and M.J.B. were employees of the GSK group of companies at the time of the study. M.J.B. and M.P. report ownership of GSK shares and/or restricted GSK shares. M.J.B., P.T.B., and M.P. are inventors named on patents and patent applications relating to meningococcal group $B$ vaccines. The remaining authors declare no competing financial interests.

Reprints and permission information is available online at http://npg.nature.com/ reprintsandpermissions/

Publisher's note: Springer Nature remains neutral with regard to jurisdictional claims in published maps and institutional affiliations.

(c) (i) Open Access This article is licensed under a Creative Commons Attribution 4.0 International License, which permits use, sharing,
adaptation, distribution and reproduction in any medium or format, as long as you give appropriate credit to the original author(s) and the source, provide a link to the Creative Commons license, and indicate if changes were made. The images or other third party material in this article are included in the article's Creative Commons license, unless indicated otherwise in a credit line to the material. If material is not included in the article's Creative Commons license and your intended use is not permitted by statutory regulation or exceeds the permitted use, you will need to obtain permission directly from the copyright holder. To view a copy of this license, visit http://creativecommons.org/ licenses/by/4.0/.

(c) The Author(s) 2018 